\title{
Modified Bagolini striated glass test: clinical applications of starlight test in binocular visual field screening
}

T Hirai, M Arai, Y Ito, M Sato

\begin{abstract}
Aim-To introduce the "starlight" test which was devised to check binocular vision in normal conditions of seeing in a rapid, easy, and cost effective manner and to estimate the possibility of its clinical use in screening the binocular visual field of patients.

Method-The Bagolini striated glass test consists of optically plano lenses with imperceptible parallel scratches that barely blur the environment but produce two perpendicular luminous stripes (right eye stripe of $45^{\circ}$ and left eye stripe of $135^{\circ}$ ) when subjects with normal binocular vision view one light source. Unlike the original Bagolini test, the starlight test uses three light sources in horizontal or vertical lines according to the testing purposes and the subject is asked to fixate upon the centre light. Through Bagolini glasses, the subject observes the resulting grid-like pattern and the state of binocular visual field of the subject can be roughly estimated.

Results-Normal subjects and patients with strabismus, visual field loss from intracranial diseases, glaucoma, retinitis pigmentosa, and functional visual loss were examined using the starlight test and findings from each case were discussed. Conclusions-The starlight test, which was made by hand at a low cost, is a simple test that can be used clinically. It provides information about the state of binocular vision of patients in normal conditions of seeing. It is also useful because it enables the examiner to share similar experiences with the examinee. The results suggest it can be effective in visual field screening.
\end{abstract}

(Br f Ophthalmol 1998;82:1288-1293)

Department of

Ophthalmology,

Nagoya University,

School of Medicine,

Nagoya, Japan

T Hirai

M Arai

Y Ito

M Sato

Correspondence to: Toshie Hirai, Department of Ophthalmology, Nagoya

University, School of

Medicine, 65 Tsuruma,

Showa-ku, Nagoya 466,

Japan.

Accepted for publication 21 April 1998 friend happened to advise her that not only visual acuity but also a visual field examination was important. It was only when the ophthalmologist performed a visual field examination at her request that her pituitary adenoma was discovered.

Further background for this study was that one of the authors (TH) who attended the community eye health course at University College London realised that in many developing countries methods of visual field examinations are mainly confrontation and tangent screen. We thought that there might be a simple and inexpensive, yet more effective, method than those mentioned. This led us to devise the starlight test to measure binocular visual field (BVF) easily and quickly using Bagolini striated glasses ${ }^{1}$.

Various methods of measuring the BVF or binocular vision of patients have been published by many researchers, especially in the field of strabismus and amblyopia..$^{2-5}$ Measurements of BVF for patients with functional visual loss, consists mainly of examining the Goldmann perimetry with both eyes open without any dissociation, have also been reported. ${ }^{67}$ The effect of restriction of BVF on driving performance has also been studied. ${ }^{8}$

In contrast with these previous studies, which focus on specific areas, we have examined various ocular conditions using the "starlight" test with the objective of using it as a screening test to detect visual field defects in patients. The results are presented here.

\section{Method}

A diagram of the handmade device and method of examination is shown in Figure 1. Nine electric bulbs of $2.5 \mathrm{~V}$ are attached to a $75 \mathrm{~cm}^{2}$ board in three equidistant rows. The points where the nine spotlights are fixed represent the nine cardinal directions of gaze. The spotlights can be switched on individually or simultaneously in any combination, according to the testing purposes. In ordinary examinations, the middle three lights are used in horizontal or vertical lines. For strabismic patients with $\mathrm{A}-\mathrm{V}$ patterns, the three lights on the upper and lower rows of lights were used. The device hangs on a wall and is portable. The size of $75 \mathrm{~cm}$ corresponds with the Hess coordimeter test. If the patient is examined at 1.4 metres distance from the test board an area of $15^{\circ}$ right, left, up, and down from the fixation point with a total $30^{\circ}$ visual field is measurable. This enables us to examine the state of interaction of both eyes in the $10^{\circ}-60^{\circ}$ range on the test board by changing the examination distance. As the eye is round, if we 

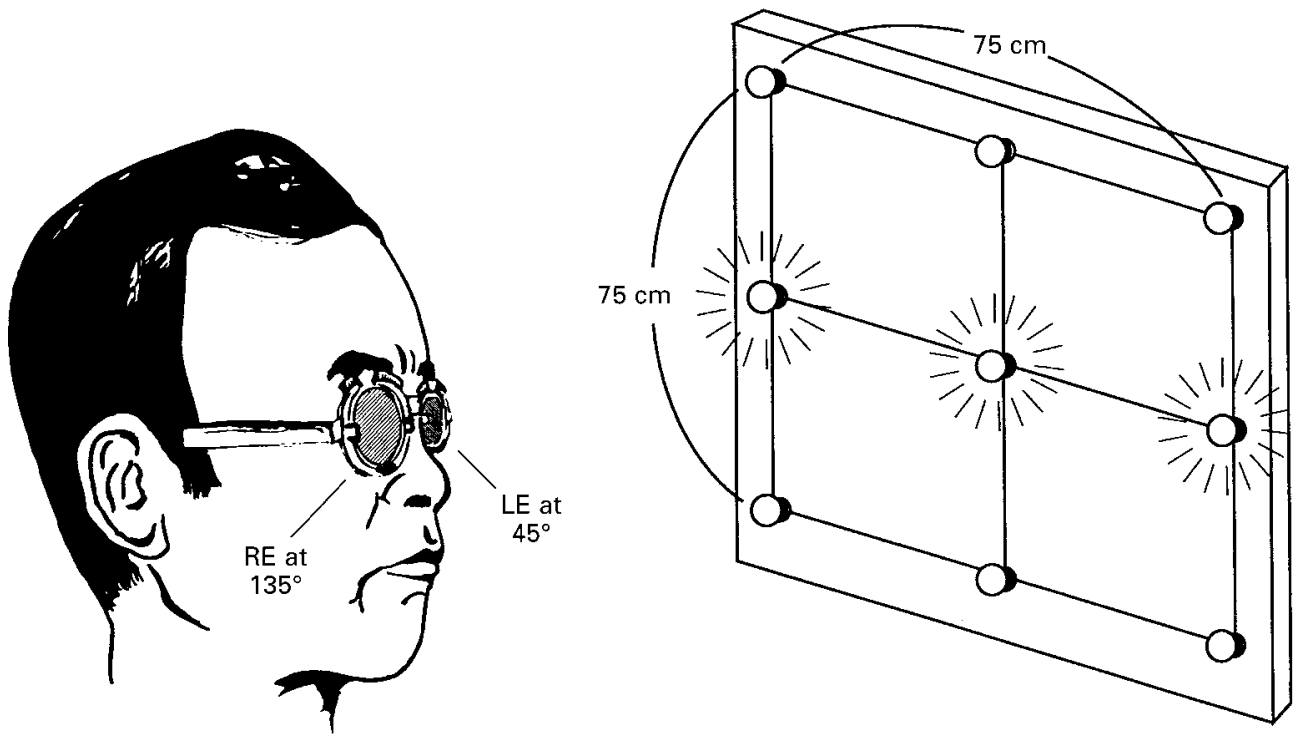

Figure 1 Diagram and method of starlight test: Bagolini glasses are placed in a trial frame in front of the corrected lenses with the axis of striation oriented at $135^{\circ}$ right eye and $45^{\circ}$ left eye.

examine the eye close to the board, the angle of visual field is not accurate but, in calculation, the relation between the examining distance and angle of visual field on the board can be determined as shown in Table 1.

There are two types of Bagolini striated glasses, No 2 and No 4 . We usually use No 4 because they produce clearer stripes. The glasses are placed in trial frame in front of the corrected lenses in such a manner that the axis of striation is oriented at $135^{\circ}$ right eye and $45^{\circ}$ left eye and the examinee perceives perpendicular luminous stripes oriented at $45^{\circ}$ right eye and $135^{\circ}$ left eye. We prefer to perform the examination in a dark room such as a perimetry room because it is easier for the patients to see the stripes, as some of them have poor visual acuity. The examination distance most often used is $140 \mathrm{~cm}\left(30^{\circ}\right)$ because the luminous stripes then extend over the board and stretch in the background to the limit that the retina can perceive, and we can estimate the entire BVF of the subjects. The subjects are asked to fixate on the centre light and to describe or draw the pattern they see. The view of a normal subject of this test is shown in Figure 2 - that is, three stripes of $45^{\circ}$ right eye and another three stripes of $135^{\circ}$ left eye (from examinee's viewpoint) with four squares created by the six stripes.

\section{Results}

NORMAL SUBJECTS

We examined 20 normal subjects with the starlight test by lowering the visual acuity of one eye using the seven different filters (Einsch-

Table 1 Relation between distance and angle of visual field

\begin{tabular}{lll}
\hline Size of screen $(\mathrm{cm})$ & Distance $(\mathrm{cm})$ & Angle of visual field $(\odot)$ \\
\hline 75 & 429 & 10 \\
75 & 213 & 20 \\
75 & 140 & 30 \\
75 & 103 & 40 \\
75 & 80 & 50 \\
75 & 65 & 60 \\
\hline
\end{tabular}

leich occlusion partielle of the Ryser Optik) originated by Bangerter. ${ }^{9}$ When one eye was $0.1(6 / 60)$ and the other eye was $1.0(6 / 6)$, all subjects recognised the difference (blurred lines and clear lines). When one eye was 0.3 and the other eye was $1.0,19$ out of 20 subjects noticed the difference. When the visual acuity of the reduced eye was better than 0.4 the results differed among individuals; some could differentiate between the blurred and clear lines while others could not. These results were similar to our previous study of the relation between stereoacuity and visual acuity. ${ }^{10}{ }^{11}$

STRABISMUS

Since Bagolini and Capobianco introduced Bagolini striated glass test (BSGT) in $1965,{ }^{1}$ it has been estimated as a simple and useful test to examine the suppression or retinal correspondence of strabismic and amblyopic patients in natural casual seeing. We acknowledged the effectiveness of the BSGT but in the course of our examinations found some significant information as follows: (1) by stimulating peripheral points of the visual field along with the fixation point, we observed that the non-dominant eye was participating in BVF in many strabismic cases, which we supposed were totally suppressed by the original test; (2) some strabismic cases showed unstable interaction of the non-dominant eye which changed from time to time. This finding has not been reported in detail in the past. An example is shown in Figure 3. (3) By comparing the results before and after surgery, we were able to estimate the expansion of the BVF of strabismic patients brought about by the effect of surgery.

VISUAL FIELD LOSS FROM INTRACRANIAL DISEASES Examination of 16 cases of visual field loss from intracranial diseases revealed $100 \%$ satisfactory results estimated by their affected diseases. Ten of them were conditions related to the pituitary gland, where bitemporal hemi- 

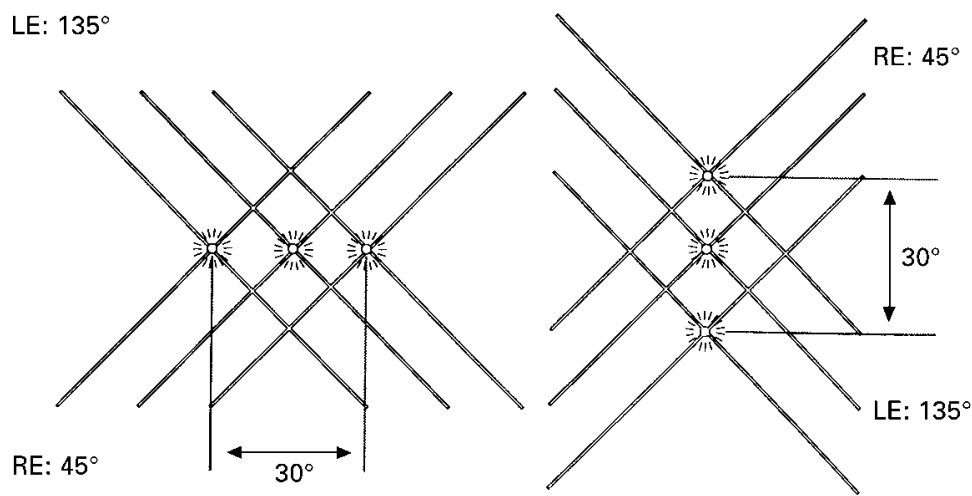

Figure 2 View of starlight test by a normal subject: the three middle horizontal light sources (left) and vertical light sources (right) were used. While fixating on the centre light through Bagolini glasses, the subject perceives the resulting grid-like pattern: three stripes of $45^{\circ}$ right eye, three stripes of $135^{\circ}$ left eye (perpendicular to the axis of the striation), and four squares created by the six stripes were observed by both eyes.
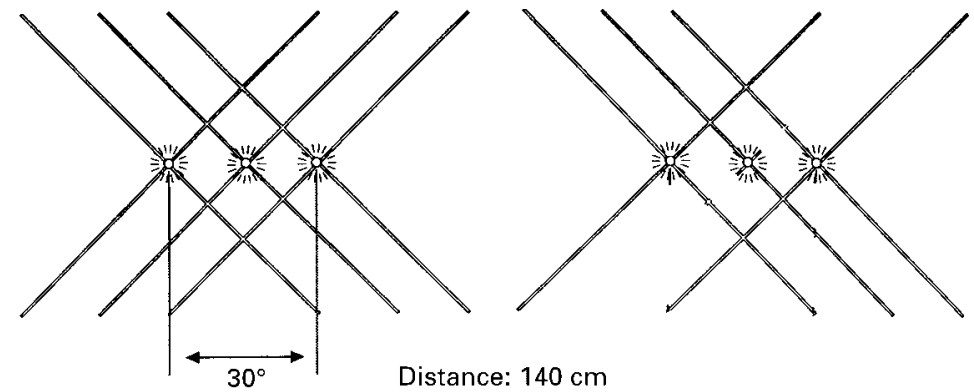

Distance: $140 \mathrm{~cm}$
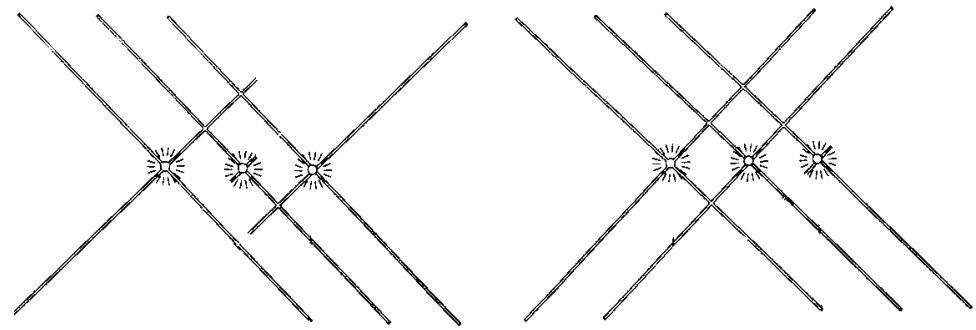

Figure 3 A case of strabismus: the patterns showed unstable interaction of the non-dominant eye (right eye) in binocular visual field which changed from time to time during 1 minute of testing.
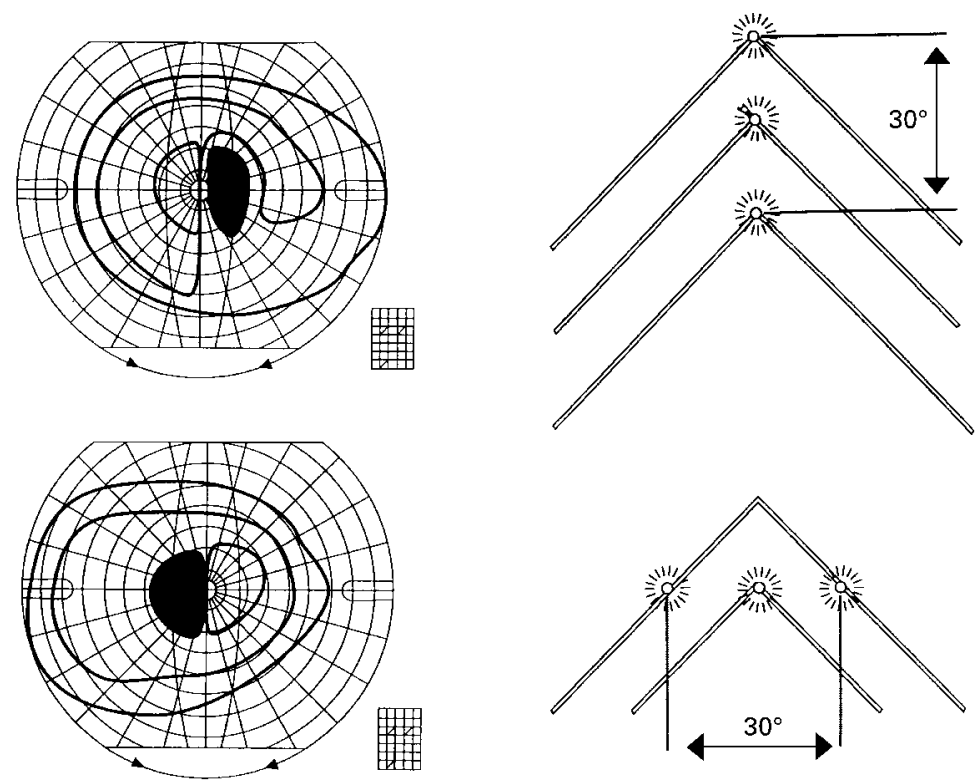

Figure 4 A case of pituitary adenoma examined by Goldmann perimetry (left) and starlight test examined in vertical and horizontal lines (at distance $140 \mathrm{cm:} 30^{\circ}$ ) (right). The starlight test showed typical bitemporal hemianopsia. anopsia was the typical symptom. The starlight test showed the same typical pattern as shown in Figure 4. For intracranial diseases, tests using the vertical column of the lights were more sensitive and interpretative than the horizontal lights, because when using the horizontal row of lights the patients with pituitary adenoma often did not notice the bottom two stripes (Fig 4, bottom right). In order to distinguish patients with homonymous and heteronymous hemianopsia from strabismus and amblyopic patients who may show similar results either as a result of suppression or reduced visual acuity in the non-dominant eye, each eye was examined separately.

\section{GLAUCOMA}

The results of 20 cases of glaucoma tested by the Humphrey 30-2, Goldmann perimetry, and the starlight test, using the horizontal rows of lights were compared. Among the tests done, the Humphrey 30-2 was most sensitive in detecting glaucoma; however, it was a boring and tiresome test and some results were misleading. Examination by Goldmann perimetry, on the other hand, was time consuming and the results were sometimes dependent on the skill of the examiner. The starlight test was not as effective as the Humphrey 30-2 in finding relative scotoma and in some cases, mainly elderly people with open angle glaucoma, could not diagnose absolute scotoma, which Goldmann perimetry identified. However, those people often indicated that while fixating on the centre light, the upper crossing point at $15^{\circ}$ from the centre where two perpendicular luminous stripes $\left(45^{\circ}\right.$ right eye and $135^{\circ}$ left eye) meet was blurred. Another interesting finding in glaucoma patients, mainly young patients with myopia, was that they indicated the stripes were irregularly broken. A typical case was a 30 year old man who also had extreme myopia in both eyes of about -16 dioptres (wearing contact lenses) who complained of misreading numbers such as eight and three because some parts of the numbers were broken (Fig 5). In this case, other areas of his visual field that seemed not affected yet when examined by both Humphrey and Goldmann perimetry revealed contrary findings with the starlight test. The patient perceived irregular stripes corresponding with these seemingly normal areas.

\section{RETINITIS PIGMENTOSA}

The 10 cases of retinitis pigmentosa examined, which were mostly of the severe form, showed results in accordance with Goldmann perimetry. One of the cases was a 37 year old man who visits our hospital every 2 years to have his visual field checked. His visual acuity in both eyes was $1.2(6 / 5)$ with myopic contact lenses and he was required to drive on occasion as part of the responsibility of his job. Lately, he felt his visual field to be getting narrow and was eager to know how different it was from those of people with normal sight. Figure 6 shows the results of his Goldmann perimetry and the starlight test. Through the starlight test, he was made aware of his visual field loss because he 

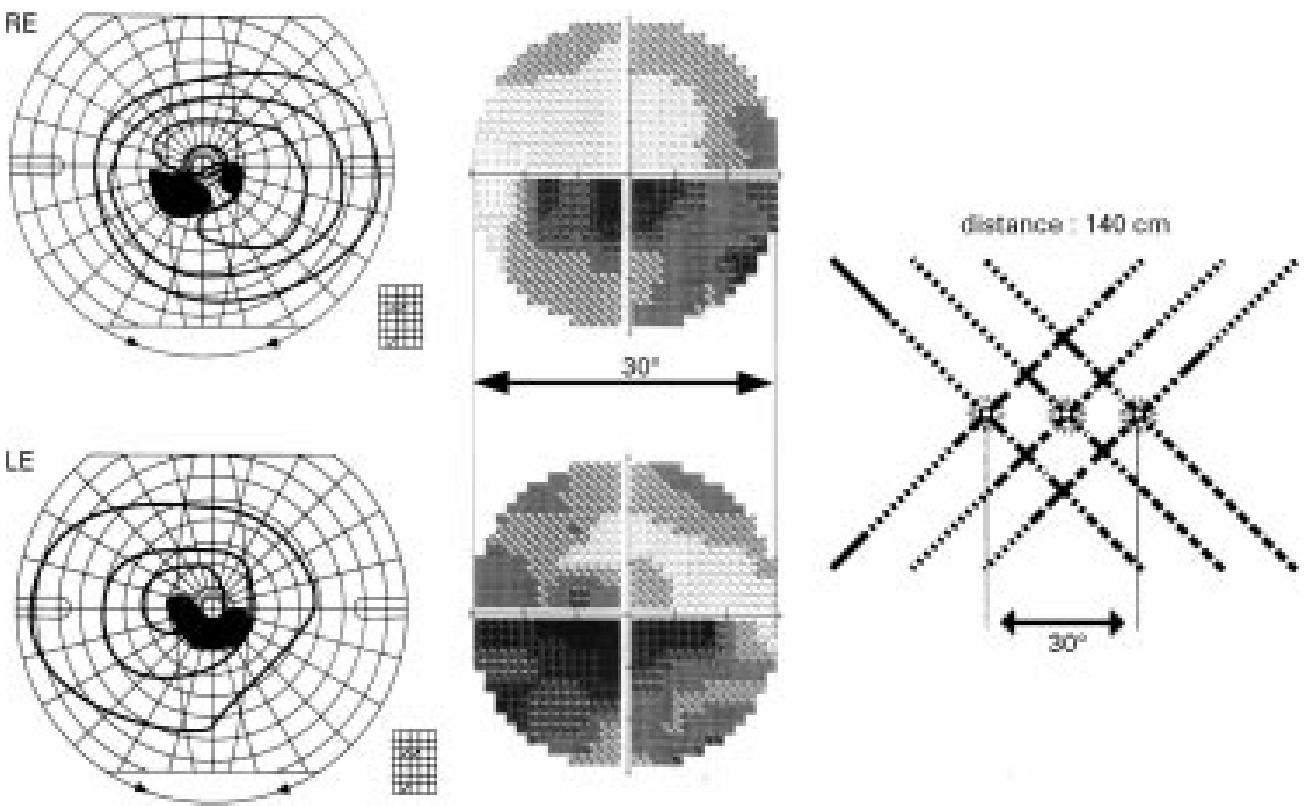

Figure 5 A case of glaucoma examined by Goldmann perimetry (left), Humphrey 30-2 (centre), and starlight test (right). In the starlight test the patient perceived irregular stripes corresponding with seemingly unaffected areas examined by Goldmann perimetry and Humphrey perimeter.

was able to see three lights and six luminous stripes at $330 \mathrm{~cm}$ from the board (about $13^{\circ}$ ).

\section{FUNCTIONAL VISUAL LOSS}

We examined 16 cases of patients diagnosed as functional visual loss, mainly schoolgirls aged 8-12 years who pointed out their visual defect at their routine school eye examination. Without any organic findings, many of them showed extreme constriction of the visual field of $10^{\circ}$ by Goldmann perimetry along with poor visual acuity. However, their drawings indicated a normal BVF corresponding to the normal pattern of the starlight test. The starlight
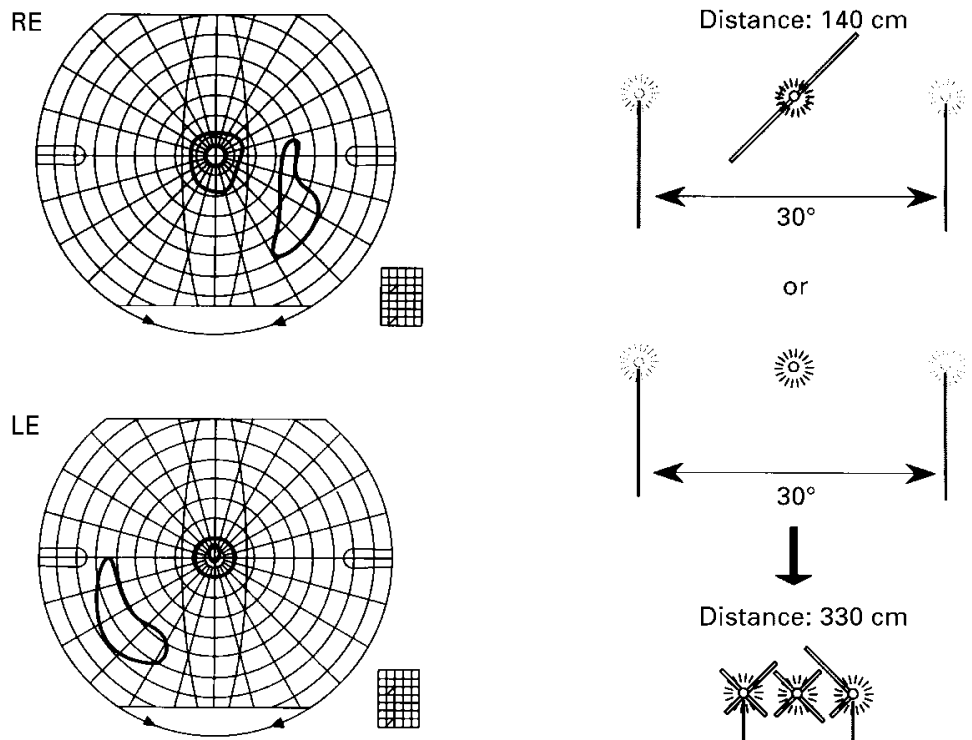

Distance: $330 \mathrm{~cm}$

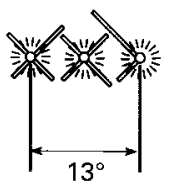

Figure 6 A case of retinitis pigmentosa examined by Goldmann perimetry (left) and starlight test (right). The patient only perceived one spotlight and a short stripe of $45^{\circ}$ at a distance of $140 \mathrm{~cm}\left(30^{\circ}\right)$ but was made aware of his visual field loss at a distance of 330 cm (about $\left.13^{\circ}\right)$. test was useful in detecting and diagnosing functional visual loss.

The starlight test was also useful in a case of unilateral visual field constriction, seen in the following patient. This 8 year old girl was referred to our hospital for presumed left optic nerve damage. According to the letter from the referring hospital, she accidentally bumped into her friend while playing ball and had her cervical vertebrae dislocated and she was still being treated by orthopaedics. Twenty days after the accident, her left eye had blurred vision. Her visual acuity was found to be 1.2 $(6 / 5)$ in both eyes and after close examination nothing in particular was found. However, her left visual field showed extreme centripetal constriction with automated perimeter and she could not walk with her right eye occluded. Figure 7 is the result of her Goldmann perimetry and starlight test, which proved the visual field of her left eye was normal as was that of the right eye.

We also examined a case of an ex-taxi driver suspected of malingering whose visual field in both eyes appeared to have been getting narrower and narrower to less than $5^{\circ}$ by Goldmann perimetry over 20 years since a traffic accident; at that time he was diagnosed as having right traumatic chorioretinitis (VA: right eye 0.02 , left eye 1.5). Using the starlight test and varying the examination distance we were able to measure his binocular visual field at more than $50^{\circ}$ by interpreting a number of his contradictory drawings. In addition, his right eye was found to be not as poor as 0.02 estimated by our results in normal subjects.

\section{Discussion}

The starlight test has three main characteristics. Firstly, in a rapid and easy manner, we can check the subject's visual acuity, visual field, and binocular vision. If a patient can see three 
RE

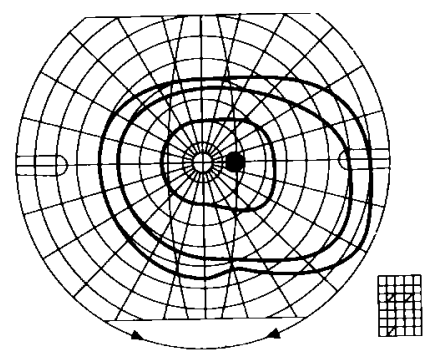

LE
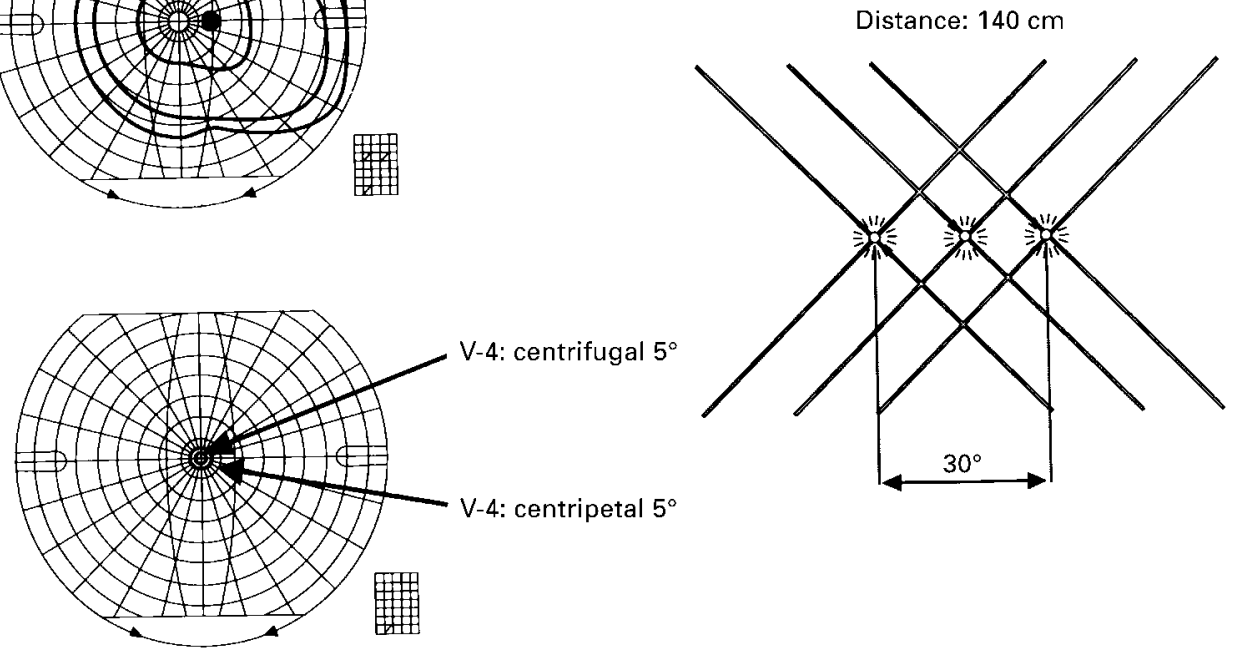

Figure 7 A case of functional visual loss. The result of Goldmann perimetry (left) showed extreme unilateral visual field constriction (left eye) but the drawing of a pattern of the starlight test by the patient proved her visual field in the left eye was normal.

spotlights and all six stripes clearly and those stripes stretch to the surrounding area we can estimate his/her visual acuity in both eyes to be good and nearly equal and his /her visual fields to be satisfactory with no significant ocular muscle imbalance.

For a person with normal single binocular vision, the examination took little time. The examination of patients with functional visual loss was also accomplished quickly, as they were able to draw a diagram of what they saw easily and rapidly.

The second characteristic is that although the result is not as precise as other perimetric methods, it is more interpretative in conveying the state of the actual visual field of patients. For strabismic patients, we observed that the non-dominant eye was unable to fixate well and moved restlessly, while the dominant eye fixated stably (Fig 3). The results of the starlight test in the case of retinitis pigmentosa had more impact than the results of Goldmann perimetry when the patient indicated he saw only one spotlight and a short stripe of $45^{\circ}$ which sometimes disappeared at a distance of $140 \mathrm{~cm}$ (Fig 6). For the cases of malingering, we can obtain documentation of self drawn visual fields.

The third advantage of the starlight test is that we can share similar experiences with the patients and their parents. We found their observations were sometimes quite keen and from these observations we have learned a lot. A patient who was examined at $80 \mathrm{~cm}$ (angle of $50^{\circ}$ ) claimed the stripes were not straight but curved and we, along with all other 20 normal subjects, experienced the same phenomenon (Fig 8, left). This phenomenon was observed even by subjects without corrected glasses and the degree of the curved line increased as we approached the board. Therefore, we concluded that this phenomenon occurs because our eyes are round and stripes, which fall on the peripheral retina, are seen as curved, as Helmholtz ${ }^{1213}$ proved in different manner in 1867 (Fig 8, left).
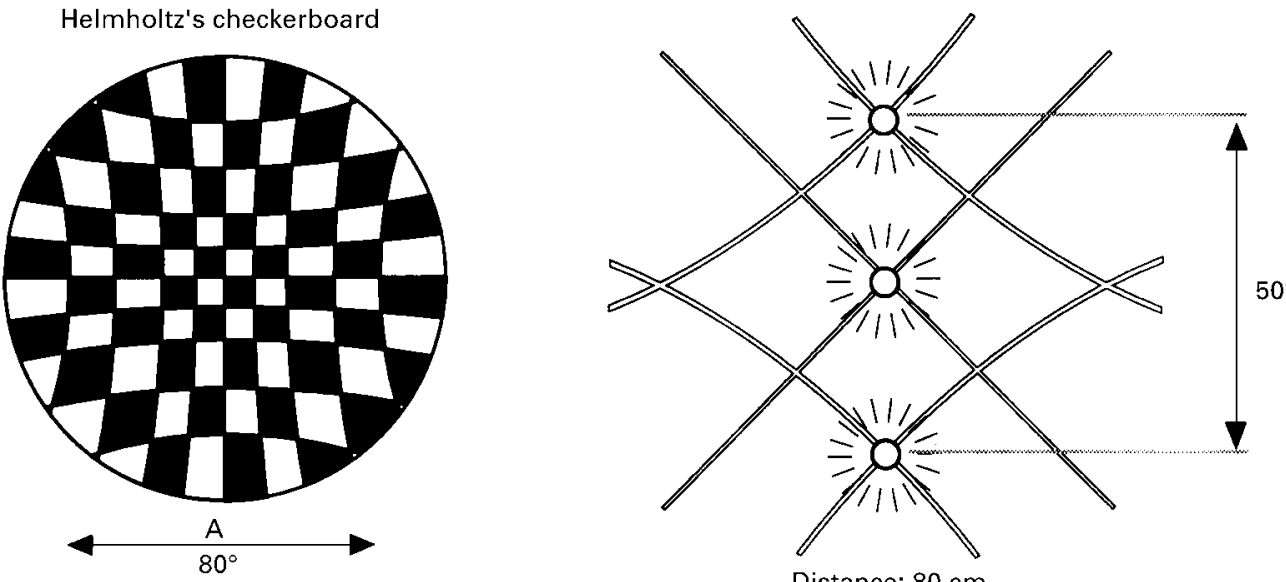

Figure 8 Left: when this checkerboard is positioned in front of one eye, at a distant of $A$ (about $80^{\circ}$ by our calculation), it appears rectilinear. Right: at a distance of $80 \mathrm{~cm}\left(50^{\circ}\right)$ a subject recognised curved stripes in the starlight test. 
The disadvantages of the test are as follows. It is useful for gross visual field loss but the difficulty lies on how to screen early defects of the visual field in glaucoma patients. Flow charts for screening must be carefully considered because this simple test sometimes produces useful but unexpected answers from patients. For example, in the case of pituitary adenoma (Fig 4: upper right), when we asked how many spotlights and stripes were seen, the patient answered three spotlights and six stripes. We thought she had normal visual field but when we asked how many squares were observed and she said none, we asked her to draw a picture of what she was observing. From her drawing, we were able to ascertain that she had bitemporal visual field loss. Although the starlight test has disadvantages, we estimate that it is more effective than confrontation and more time saving than tangent screen.

In our department, where expensive new instruments compete for valuable space, this simple handmade starlight test has proved indispensable. The stripes produced by light sources, like a constellation of stars in the sky, simply indicate the enchanting mechanism of our BVF; which was why we have succeeded in stimulating the minds of many patients with functional visual loss and have been able to elicit results from them.

The authors gratefully acknowledge Professor Yozo Miyake for his inspiring encouragement and Dr F P Inocencio for his useful comments.

1 Bagolini B, Capobianco NC. Subjective space in comitant squint. Am 7 Ophthalmol 1965;15:430-42.

2 Pratt-Jonson JA, MacDonald AL. Binocular visual field in strabismus. Can $\mathcal{f}$ Ophthalmol 1976;11:37-41.

3 Campos EC. Binocularity in comitant strabismus: binocula visual fields studies. Doc Ophthalmol 1982;53:249-81.

4 Herzau V. Aspect of sensory adaptation in strabismus. Arch Ophthalmol 1983;101:218-24.

5 Nuzzi G, Cantu C, Camparini M. Second generation binocular polaroid test. $\mathcal{F}$ Pediatr Ophthalmol Strabismus 1996;33:235-40

6 Keane JR. Hysterical hemianopsia. The "missing half" field deft. Arch Ophthalmol 1979;97:865-6.

7 Gittinger JW. Functional monocular temporal hemianopsia. Am f Ophthalmol 1986;101:226-31.

8 Wood JM, Troutbeck R. Effect of restriction of the binocular visual field on driving performance. Ophthalmic Physiol Optics 1992;12:291-8.

9 Bangerter A. Die Okklusion in der Pleoptik und Orthoptik. Klin Monatsbl Augenheilkd 1960;136:305-31.

10 Hirai T, Yonekura Y. Suppression in anisometropic ambliopia. Fap Orthopt F 1973;1:15.

1 Hirai T. Binocular vision tests for near. In: Practical ophthalmology. Vol 18. 1st ed. Tokyo: Bunkodo, 1995:144-8.

2 Helmholtz H. Helmholtz's treatise on physiological optics. Translation from the third German ed. Southall JPC, ed. Vol III. The perceptions of vision. New York: Optic Society of America, 1925:154-232.

13 Leiliu CMS. Functional division of human retina. V. In: Lakshminrarayanan, ed. Basic and clinical applications of vision. Amsterdam: Kluwer, 1997:295-8. 